\title{
Universiteit
}

Leiden

The Netherlands

\section{Development of the high-temperature phase of hexagonal manganites}

Lonkai, T.; Tomuta, D.G.; Amann, U.; Ihringer, J.; Hendrikx, R.W.A.; Többens, D.M.; Mydosh, J.A.

\section{Citation}

Lonkai, T., Tomuta, D. G., Amann, U., Ihringer, J., Hendrikx, R. W. A., Többens, D. M., \& Mydosh, J. A. (2004). Development of the high-temperature phase of hexagonal manganites. Physical Review B, 69(13), 134108. doi:10.1103/PhysRevB.69.134108

Version: $\quad$ Not Applicable (or Unknown)

License: $\quad$ Leiden University Non-exclusive license

Downloaded from: https://hdl.handle.net/1887/77091

Note: To cite this publication please use the final published version (if applicable). 


\title{
Development of the high-temperature phase of hexagonal manganites
}

\author{
Th. Lonkai, ${ }^{1, *}$ D. G. Tomuta, ${ }^{2, \dagger}$ U. Amann, ${ }^{1}$ J. Ihringer, ${ }^{1}$ R. W. A. Hendrikx, ${ }^{2}$ D. M. Többens, ${ }^{3}$ and J. A. Mydosh ${ }^{2,4}$ \\ ${ }^{1}$ Institut für Angewandte Physik, Universität Tübingen, Auf der Morgenstelle 10, 72076 Tübingen, Germany \\ ${ }^{2}$ Kamerlingh Onnes Laboratory, Leiden University, P.O. Box 9504, 2300 RA Leiden, The Netherlands \\ ${ }^{3}$ BENSC, HMI Berlin, Glienicker Straße 100, 14109 Berlin, Germany \\ ${ }^{4}$ Max-Planck Institut für Chemische Physik Fester Stoffe, Nöthnitzer Straße 40, 01187 Dresden, Germany
}

(Received 14 October 2003; published 19 April 2004)

\begin{abstract}
Reports about the ferroelectric ordering temperatures in the multiferroic hexagonal $R \mathrm{MnO}_{3}$ system are controversial: transition temperatures varying between $\approx 900 \mathrm{~K}$ and $\approx 1300 \mathrm{~K}$ are reported for the same material. To elucidate the structural changes leading to ferroelectric distortions in hexagonal manganites, we calculate the irreducible representations of the distortions from the possible high-temperature symmetry $P 6_{3} / \mathrm{mmc}$ to the low-temperature symmetry $\mathrm{Pb}_{3} \mathrm{~cm}$. There are four different orthogonal modes, of which only one allows a spontaneous electric polarization. Structure refinements and an accurate statistical analysis of neutron powder-diffraction data of $\mathrm{TmMnO}_{3}$, based on this group-theoretical analysis, reveal two phase transitions: We extrapolate a polar to nonpolar transition temperature of $T_{n p t}=1433(27) \mathrm{K}$, where the hexagonal bitetrahedra start to tilt, while the ferroelectric distortion appears at $T_{F E}=1050(50) \mathrm{K}$. For $R=\mathrm{Lu}, \mathrm{Yb}$ the tilt of the bitetrahedra and the buckling of the $R$ layers as well as the ferroelectric distortion were extrapolated to comparable temperatures.
\end{abstract}

DOI: 10.1103/PhysRevB.69.134108

PACS number(s): 77.80.Bh, 61.12.Ld, 61.10.Nz, 02.20.-a

\section{INTRODUCTION}

Multiferroic compounds of hexagonal manganites $R \mathrm{MnO}_{3}(R=\mathrm{Er}, \mathrm{Ho}, \mathrm{In}, \mathrm{Lu}, \mathrm{Sc}, \mathrm{Tm}, \mathrm{Y}, \mathrm{Yb})$ represent a challenging and fascinating field in solid-state physics. The simultaneous presence of electric and magnetic ordering $\left(T_{F E}\right.$ $\left.\approx 1000 \mathrm{~K}, T_{N} \approx 100 \mathrm{~K}\right),{ }^{1}$ the famous problem with two nearly homometric magnetic lattices ${ }^{2}$ and the unusual strong magnetic two-dimensional (2D) short-range order, ${ }^{3}$ the existence of ferroelectromagnetic domains, ${ }^{4}$ and the coupling of electric and magnetic order parameters ${ }^{5}$ attracted interest of experimentalists, engineers and theoreticians. Possible applications as random access memory devices are proposed according to the ferromagnetic and ferroelectric behaviors of epitaxial thin films. ${ }^{6,7}$ Though systematic studies on this class started in the mid 1960s, 2,8 and recent publications are numerous, e.g., Refs. 9-12, the magnetic structure remained controversial until optical measurements were applied ${ }^{13}$ and a novel statistical method of data analysis for diffraction data was developed. ${ }^{14}$

The analysis of the electric ordering process is likewise problematic. Hexagonal manganites order in the space group (SG) $P 6_{3} \mathrm{~cm} .{ }^{8}$ The absence of the inversion center in $P 6_{3} \mathrm{~cm}$ allows off-center displacements along the polar axis and by this a spontaneous electric polarization. Furthermore, local-spin-density approximations (LSDA) based on the paraelectric space group $P 6_{3} / m m c$ showed that ferroelectric displacements in hexagonal manganites will only be favored along the polar axis. ${ }^{15}$ LSDA were able to achieve even more remarkable results, e.g., $\mathrm{YMnO}_{3}$ is correctly described as an insulator, ${ }^{16,17}$ thus LSDA studies of the ferroelectric displacements are now possible.

In recent publications the origin of the ferroelectric distortion in hexagonal manganites is suggested to be generated by a temperature-dependent tilt of the $\mathrm{MnO}_{5}$ coordination polyhedra and a buckling of the $R$ layers, ${ }^{18}$ indicating a structural phase transition from the nonpolar SG $\mathrm{Pb}_{3} / \mathrm{mmc}$ (Ref. 19) $\left(\overline{1} \in P 6_{3} / \mathrm{mmc}\right)$ to the polar $\mathrm{SG} P 6_{3} \mathrm{~cm}$ $\left(\overline{1} \notin P 6_{3} \mathrm{~cm}\right)$. This agrees with early conjectures by Lukaszewicz and Karat-Kalicinska. ${ }^{20}$

However, macroscopic measurements seem to contradict the results from diffraction data: for $\mathrm{YMnO}_{3}, T_{F E}=920 \mathrm{~K}$ detected by direct measurement of the pyroelectric current ${ }^{21}$ is several hundred kelvins lower than the reported structural phase transition at about $1270 \mathrm{~K}^{22}$ obtained via x-ray diffraction. Table I lists the ferroelectric to nonferroelectric transition temperatures $\left(T_{F E}\right)$ as collected by Smolenskii and Chupis $^{22}$ in comparison to the structural transition temperatures, estimated by Abrahams ${ }^{19}\left(T_{n p t}\right)$. Note here the significant differences. Despite the successes of experimental and computational physics, the electric ordering process is still not understood - the polyhedral tilting and the ferroelectric distortion occur at different temperatures.

In this manuscript we present a detailed analysis of the development of the high-temperature structure of hexagonal manganites. Calculations of the irreducible presentation of the high-temperature structure explain the necessity of two different transition temperatures $T_{F E}$ and $T_{n p t}$, as a ferroelectric phase transition with a nonzero propagation vector contradicts Landau's theory of structural phase transitions. Rietveld refinements and a careful statistical analysis of x-ray- and neutron-diffraction data of $R \mathrm{MnO}_{3}$-powdered samples, $R=\mathrm{Lu}, \mathrm{Tm}, \mathrm{Yb}$, reveal indeed two distinct transitions $T_{F E}$ and $T_{n p t}$. We explain the tripling of the unit cell at $T_{n p t}$ by a tilt of the fivefold coordination polyhedron of $\mathrm{Mn}$ and a corrugation of the $R$ layers, while the ferroelectric phase transition at $T_{F E}$ is generated without change of symmetry by a displacement of the oxygen and manganese ions within the fivefold coordination polyhedron of $\mathrm{Mn}$.

The present work represents the results of a cooperation between the Kamerlingh-Onnes Laboratory, Leiden Univer- 
TABLE I. Transition temperatures for the hexagonal $R \mathrm{MnO}_{3}$ compounds. $T_{F E}$ denotes the transition temperature from ferroelectric to nonferroelectric state (Ref. 22), and $T_{n p t}$ denotes the structural transition temperature from a high symmetry, e.g., $P 6_{3} / m m c$ to the low symmetry $\mathrm{Pb}_{3} \mathrm{~cm}$ as estimated by Abrahams (Ref. 19).

\begin{tabular}{lcc}
\hline \hline Compound & $T_{F E}(\mathrm{~K})$ & $T_{n p t}(\mathrm{~K})$ \\
\hline $\mathrm{YMnO}_{3}$ & 920 & $\approx 1220$ \\
$\mathrm{LuMnO}_{3}$ & $>750$ & $\approx 1290$ \\
$\mathrm{YbMnO}_{3}$ & 993 & $\approx 1270$ \\
$\mathrm{ScMnO}_{3}$ & & $\approx 1220$ \\
$\mathrm{TmMnO}_{3}$ & $>573$ & \\
$\mathrm{ErMnO}_{3}$ & 833 & \\
$\mathrm{HoMnO}_{3}$ & 873 & \\
\hline \hline
\end{tabular}

sity (sample preparation, x-ray powder diffraction, neutron powder diffraction, Rietveld refinements) and the Institut für Angewandte Physik, Universität Tübingen (Rietveld refinements, statistical analysis, irreducible representations). The neutron powder diffraction was performed at the E9 spectrometer, Hahn-Meitner Institut, Berlin.

\section{NUCLEAR STRUCTURE}

The nuclear structure of hexagonal manganites at room temperature, SG $P 6_{3} \mathrm{~cm}$, is well known, ${ }^{8,23-26}$ see Table II. Hexagonal manganites are formed from layers of $2 \mathrm{D}$ connected distorted and tilted $\mathrm{MnO}_{5}$ polyhedra in an unusual fivefold coordination, which are separated by corrugated $R$ layers, see Fig. 1.

To gain better insight in the evolution of the nuclear phases, it is useful to begin the symmetry analysis with the highest possible symmetry, the so-called aristotype. ${ }^{28}$ Related structures of lower symmetry are called hettotypes. The obvious aristotype of hexagonal manganites, as suggested by Abrahmas $^{19}$ as a possible nonpolar high-temperature SG of hexagonal manganites, is $P 6_{3} / m m c$ (Table III). The next step is a comparison to its hettotype $P 6_{3} \mathrm{~cm}$, the lowtemperature structure of hexagonal manganites.

We indicate the crystal axes of the high- and low-
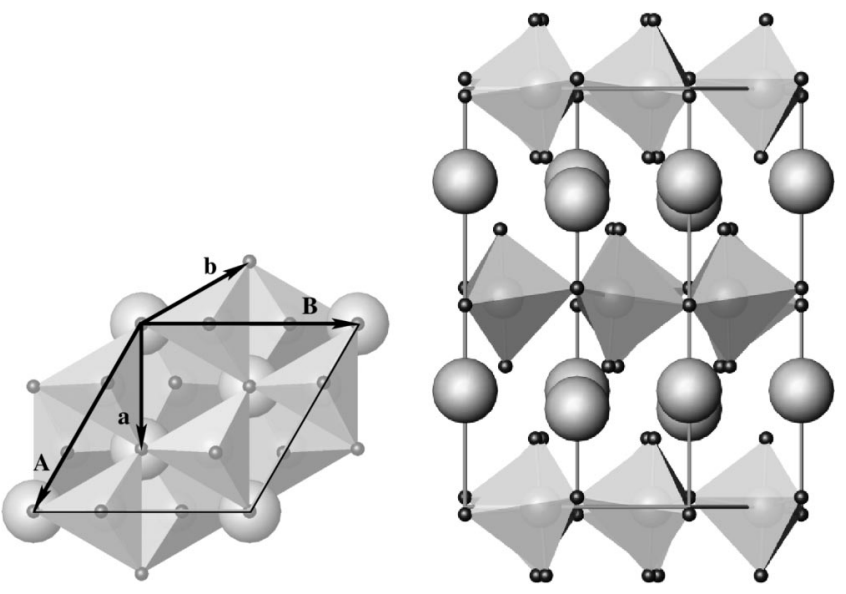

FIG. 1. Hexagonal $R \mathrm{MnO}_{3}$ at room temperature, SG $P 6_{3} \mathrm{~cm}$, $(A B)$ plane (left), $(B C)$ plane (right), big spheres $R$, small spheres $\mathrm{O}, \mathrm{Mn}$ lies in the middle of the coordination polyhedra. In addition the hexagonal axes of the low-temperature phase $P 6_{3} \mathrm{~cm} \vec{A}, \vec{B}$ and of the high-temperature phase $P 6_{3} / m m c, \vec{a}, \vec{b}$ are depicted (left).

temperature phases as $\vec{a}, \vec{b}, \vec{c}$, and $\vec{A}, \vec{B}, \vec{C}$, respectively. With

$$
\sqrt{3} a \approx A, \quad \sqrt{3} b \approx B, \quad c \approx C,
$$

the hexagonal axes $\vec{a}$ and $\vec{A}$ of both structures include an angle of $\varphi=30^{\circ}$ as shown in Fig. 1, (AB) plane. The atomic positions of each SG as well as the relations between them are indicated in the fifth columns of Tables II and III.

\section{IRREDUCIBLE REPRESENTATIONS}

According to Landau's theory of structural phase transitions, any symmetry reduction is a linear combination of atomic displacements that transform as irreducible representations of the high-symmetry group.

As the unit cell is tripled, we look for a displacement for each atom $\vec{D}\left(\vec{r}_{0}\right) \in \mathrm{C}^{3}$,

$$
\vec{D}(\vec{r})=\operatorname{Re}\left[\vec{D}\left(\vec{r}_{0}\right) \exp (2 \pi i \vec{k} \vec{\Delta} r)\right], \quad \vec{\Delta} r=\vec{r}-\vec{r}_{0}
$$

TABLE II. Low-temperature SG $\mathrm{P}_{3} \mathrm{~cm}$ of hexagonal manganites, atom type, Wyckoff notation, site symmetry, and atomic position. In the fifth column the corresponding atomic positions of the possible high-temperature phase (Ref. 19) are given. Values not given in decimal notation are fixed by symmetry. As the structure is polar, the $z$ position of one atom, usually $\mathrm{Mn}$, is fixed to zero. Approximations for the atomic positions (Refs. 8, 14, and 23-26) or for isostructural $\mathrm{YGaO}_{3}$ (Ref. 27) are given, but values may vary for different $R$ and different temperatures.

\begin{tabular}{lcccc}
\hline \hline $\mathrm{Mn}$ & $6(c)$ &.$m$ & $(0.335,0,0.000)$ & $(1 / 3,0,0)$ \\
$R(1)$ & $2(a)$ & $3 . m$ & $(0,0,0.27)$ & $(0,0,1 / 4)$ \\
$R(2)$ & $4(b)$ & $3 .$. & $(1 / 3,2 / 3,0.235)$ & $(1 / 3,2 / 3,1 / 4)$ \\
$\mathrm{O}(1)$ & $6(c)$ &..$m$ & $(0.31,0,0.167)$ & $(1 / 3,0,0.167)$ \\
$\mathrm{O}(2)$ & $6(c)$ &..$m$ & $(0.36,0,-0.167)$ & $(1 / 3,0,-0.167)$ \\
$\mathrm{O}(3)$ & $2(a)$ & $3 . m$ & $(0,0,-0.02)$ & $(0,0,0)$ \\
$\mathrm{O}(4)$ & $4(b)$ & $3 .$. & $(1 / 3,2 / 3,0.015)$ & $(1 / 3,2 / 3,0)$ \\
\hline \hline
\end{tabular}


TABLE III. Possible high-temperature SG $\mathrm{P}_{3} / \mathrm{mmc}$ of hexagonal manganites (Ref. 19), atom type, Wyckoff notation, site symmetry, and atomic position. In the fifth column the corresponding atoms of the low-temperature phase are given. Values not given in decimal notation are fixed by the symmetry. The value for $z\left(\mathrm{O}_{a p}\right)$ is taken from Abrahams (Ref. 19).

\begin{tabular}{lcccc}
\hline \hline $\mathrm{Mn}$ & $2(d)$ & $\overline{6} m 2$ & $(1 / 3,2 / 3,3 / 4)$ & $\mathrm{Mn}$ \\
$R$ & $2(a)$ & $\overline{3} m$ & $(0,0,0)$ & $R(1), R(2)$ \\
$\mathrm{O}_{a p}$ & $4(f)$ & $3 m$ & $(1 / 3,2 / 3,0.917)$ & $\mathrm{O}(1), \mathrm{O}(2)$ \\
$\mathrm{O}_{e q}$ & $2(b)$ & $\overline{6} m 2$ & $(0,0,1 / 4)$ & $\mathrm{O}(3), \mathrm{O}(4)$ \\
\hline \hline
\end{tabular}

leading from the high-temperature symmetry $P 6_{3} / m m c$ to the low-temperature symmetry $P 6_{3} \mathrm{~cm}$.

Here, $\operatorname{Re}\left[\vec{D}\left(\vec{r}_{0}\right)\right]$ denotes the distortion of the original unit cell in $P 6_{3} / m m c$. The propagation vector $\vec{k} \in \mathbb{R}^{* 3}, \vec{k}$ $=(1 / 3,1 / 3,0)$, as can be calculated easily, modulates this distortion, tripling the unit cell. There are four subgroups of $\mathrm{P6}_{3} / \mathrm{mmc}$, which are supergroups of $\mathrm{P6}_{3} \mathrm{~cm}$ :

$$
\left\{P 6_{3} / m m c, P 6_{3} m c, P 6_{3} / m c m, P 6_{3} c m\right\} \text {. }
$$

Using the program ISOTROPY, ${ }^{29}$ based on Ref. 30, we derive two one-dimensional modes $\Gamma_{1}^{+}: P 6_{3} / m m c \Rightarrow P 6_{3} / m m c$ and $\Gamma_{2}^{-}: P 6_{3} / m m c \Rightarrow P 6_{3} m c$ and two two-dimensional modes $\quad K_{1}: \quad P 6_{3} / m m c \Rightarrow P 6_{3} / m c m$ and $K_{3}$ : $P 6_{3} / m m c \Rightarrow P 6_{3} c m$, describing the distortions $\vec{\Delta}(X)$ of atom $X$ (Ref. 31$)$, see Table IV.

(1) The full symmetric or so-called breathing mode $\Gamma_{1}^{+}$: $P 6_{3} / m m c \Rightarrow P 6_{3} / m m c$, with the refineable order parameter $\lambda_{1}\left(\mathrm{O}_{a p}\right)$,

$$
\vec{\Delta}(\mathrm{O}(1))=-\vec{\Delta}(\mathrm{O}(2))=\lambda_{1}\left(\mathrm{O}_{a p}\right) \vec{C}
$$

(2) The proper ferroelectric mode $\Gamma_{2}^{-}$: $P 6_{3} / m m c \Rightarrow P 6_{3} m c$, with the refineable order parameters $\lambda_{2}(\mathrm{Mn}), \lambda_{2}(R), \lambda_{2}\left(\mathrm{O}_{e q}\right)$, and $\lambda_{2}\left(\mathrm{O}_{a p}\right)$,

$$
\begin{gathered}
\vec{\Delta}(\mathrm{Mn})=\lambda_{2}(\mathrm{Mn}) \vec{C}, \\
\vec{\Delta}(R(1))=\vec{\Delta}(R(2))=\lambda_{2}(R) \vec{C},
\end{gathered}
$$

\begin{tabular}{|c|c|c|c|c|}
\hline Mode & Volume & SG & Type & $\vec{k}$ \\
\hline$\Gamma_{1}^{+}$ & 1 & $\begin{array}{l}P 6_{3} / m m c \\
(194) D_{6 h}^{4}\end{array}$ & $\begin{array}{c}\text { Full } \\
\text { symmetric }\end{array}$ & $(0,0,0)$ \\
\hline$\Gamma_{2}^{-}$ & 1 & $\begin{array}{c}P 6_{3} m c \\
(186) C_{6 v}^{4}\end{array}$ & $\begin{array}{c}\text { Proper } \\
\text { ferroelectric }\end{array}$ & $(0,0,0)$ \\
\hline$K_{1}$ & 3 & $\begin{array}{l}P 6_{3} / m c m \\
(193) D_{6 h}^{3}\end{array}$ & $\begin{array}{c}\text { Non } \\
\text { ferroic }\end{array}$ & $(1 / 3,1 / 3,0)$ \\
\hline$K_{3}$ & 3 & $\begin{array}{c}P 6_{3} \mathrm{~cm} \\
(185) C_{6 v}^{3}\end{array}$ & $\begin{array}{c}\text { Improper } \\
\text { ferroelectric }\end{array}$ & $(1 / 3,1 / 3,0)$ \\
\hline
\end{tabular}

TABLE IV. The possible high-temperature phase transition $\mathrm{PG}_{3} / \mathrm{mmc}$ to $\mathrm{PG}_{3} \mathrm{~cm}$ in terms of irreducible representations (Refs. 29 and 30).

$$
\begin{aligned}
& \vec{\Delta}(\mathrm{O}(1))=\vec{\Delta}(\mathrm{O}(2))=\lambda_{2}\left(\mathrm{O}_{a p}\right) \vec{C} \\
& \vec{\Delta}(\mathrm{O}(3))=\vec{\Delta}(\mathrm{O}(4))=\lambda_{2}\left(\mathrm{O}_{e q}\right) \vec{C}
\end{aligned}
$$

leading to a ferroelectric distortion. ${ }^{32}$

(3) The nonferroic mode $K_{1}: P 6_{3} / m m c \Rightarrow P 6_{3} / m c m$, with refineable order parameters $\lambda_{3}(\mathrm{Mn})$ and $\lambda_{3}\left(\mathrm{O}_{a p}\right)$,

$$
\vec{\Delta}(\mathrm{Mn})=\lambda_{3}(\mathrm{Mn}) \vec{A}
$$

$$
\vec{\Delta}(\mathrm{O}(1))=\vec{\Delta}(\mathrm{O}(2))=\lambda_{3}\left(\mathrm{O}_{a p}\right) \vec{A}
$$

leading to a displacement of the $\mathrm{O}(1)-\mathrm{Mn}-\mathrm{O}(2)$ axis of the $\mathrm{MnO}_{5}$ bitetrahedra parallel to $\vec{A}$.

(4) Finally, the improper ferroelectric mode $K_{3}$ : $P 6_{3} / m m c \Rightarrow P 6_{3} \mathrm{~cm}$, with refineable order parameters $\lambda_{4}(R), \lambda_{4}\left(\mathrm{O}_{e q}\right)$, and $\lambda_{4}\left(\mathrm{O}_{a p}\right)$,

$$
\begin{gathered}
\vec{\Delta}(R(1))=-\vec{\Delta}(R(2))=\lambda_{4}(R) \vec{C} \\
\vec{\Delta}(\mathrm{O}(1))=-\vec{\Delta}(\mathrm{O}(2))=\lambda_{4}\left(\mathrm{O}_{a p}\right) \vec{A} \\
\vec{\Delta}(\mathrm{O}(3))=-\vec{\Delta}(\mathrm{O}(4))=\lambda_{4}\left(\mathrm{O}_{e q}\right) \vec{C}
\end{gathered}
$$

leading to a tilt of the $\mathrm{MnO}_{5}$ bitetrahedra and a corrugation of the $R$ layers.

It is important to realize that though $K_{3}$ leads directly to the nonpolar SG $\mathrm{Pb}_{3} \mathrm{~cm}$ and allows displacements parallel to $\vec{C}$, a spontaneous electric polarization can only be activated by a second mode: Let $\vec{E}$ be the sum of the displacements of an atom at the position $\vec{r}_{0}$ due to the $K_{3}$ : $P 6_{3} / m m c \Rightarrow P 6_{3} c m$ mode, summarized over the tripled unit cell of the SG $P 6_{3} \mathrm{~cm}$. Then $\vec{E}$ can be written as

$$
\vec{E}=\operatorname{Re}\left[\sum_{j=0}^{2} \vec{D}\left(\vec{r}_{0}\right) \exp \left(\frac{2 \pi i}{3} j\right)\right]=(0,0,0)
$$

as $\exp (2 / 3 \pi i)$ denotes the third complex root of -1 . In fact any phase transition from a nonpolar SG to a polar SG with a propagation vector $\vec{k} \neq(0,0,0)$ cannot lead to a spontaneous electric polarization.

Thus the discrepancy between the transition temperatures obtained by measurements of the pyroelectric current and $\mathrm{x}$-ray powder diffraction were to be expected. While cooling, a peak in the pyroelectric current indicates the freezing-out of the proper ferroelectric mode, while the appearance of new peaks in x-ray powder-diffraction patterns indicates the tripling of the unit cell. The latter contradicts a proper ferroelectric mode, and different modes are usually correlated to different energies, and, thus, transition temperatures.

Figure 2 is a diagram of the group-subgroup relations between the nuclear SG $P 6_{3} / m m c$ and $P 6_{3} \mathrm{~cm}$ in terms of irreducible representations. The diagram is depicted as a two-step phase transition, because two modes are always required to explain a distortion of a nonferroic crystal obeying 


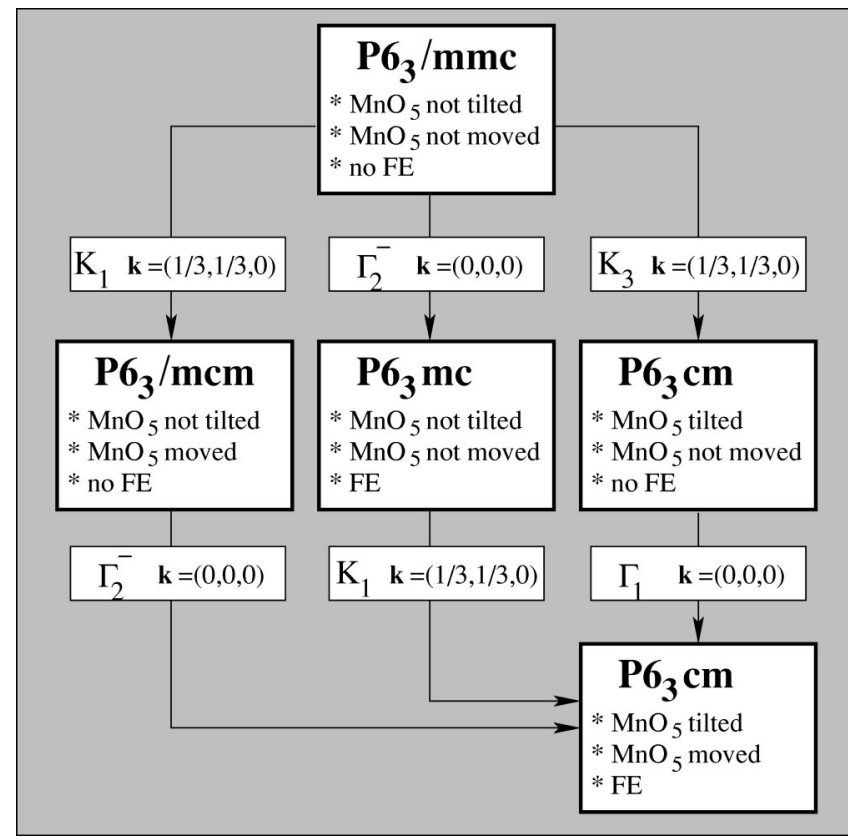

FIG. 2. There are two modes required to explain a distortion of the possible high-temperature phase of hexagonal manganites $\mathrm{P6}_{3} / \mathrm{mmc}$ (Ref. 19) to the known low-temperature structure of hexagonal manganites (Refs. 23-26), a crystal obeying the symmetry $P 6_{3} \mathrm{~cm}$ with a tilted bitetrahedron with its axis displaced from the high-symmetric position of $x(\mathrm{Mn})=1 / 3$, and with a ferroelectric distortion.

the symmetry $P 6_{3} / m m c$ to a ferroelectric crystal obeying the symmetry $P 6_{3} \mathrm{~cm}$. The three routes are as follows.

(1) The $K_{1}: P 6_{3} / m m c \Rightarrow P 6_{3} / m c m$ mode displaces the $\mathrm{O}_{a p}-\mathrm{Mn}-\mathrm{O}_{a p}$ axis of the $\mathrm{MnO}_{5}$ bitetrahedron from its highsymmetric position without tilting it and triples the unit cell, is followed by the $\Gamma_{2}^{-}: P 6_{3} / \mathrm{mcm} \Rightarrow P 6_{3} \mathrm{~cm}$ mode, which tilts the bitetrahedra, corrugates the $R$ layers, and generates a ferroelectric distortion.

(2) The $\Gamma_{2}^{-}: P 6_{3} / m m c \Rightarrow P 6_{3} m c$ mode, which generates a ferroelectric distortion, is followed by the $K_{1}$ : $P 6_{3} m c \Rightarrow P 6_{3} \mathrm{~cm}$ mode, tilting the bitetrahedra, moving them from their high-symmetric positions, corrugates the $R$ layers, and triples the unit cell.

(3) The $K_{3}: P 6_{3} / m m c \Rightarrow P 6_{3} c m$ mode tilts the $\mathrm{MnO}_{5}$ bitetrahedra, without displacing them, corrugates the $R$ layers and triples the unit cell, is followed by the $\Gamma_{1}$ : $P 6_{3} \mathrm{~cm} \Rightarrow P 6_{3} \mathrm{~cm}$ mode, which displaces the $\mathrm{O}(1)-\mathrm{Mn}-\mathrm{O}(2)$ axis of the $\mathrm{MnO}_{5}$ bitetrahedra and generates the ferroelectric distortion. ${ }^{33}$

We expect order parameters $\lambda_{i}(X)(T)$ of the same mode $j$ to have the same temperature dependence $\Lambda_{j}(T)$, independent of atom $X$. Thus, measuring the temperature dependence of the order parameters will indicate the correct sequence of phase transitions:

$$
\begin{gathered}
\forall X \exists \Lambda_{1}(T) \exists \Lambda_{2}(T): \\
\lambda_{3}(X)(T) \propto \Lambda_{1}(T), \\
\lambda_{2}(X)(T) \propto \lambda_{4}(T) \propto \Lambda_{2}(T)
\end{gathered}
$$

supports the first scenario in Fig. 2,

$$
\begin{gathered}
\forall X \exists \quad \Lambda_{1}(T) \exists \Lambda_{2}(T): \\
\lambda_{2}(X)(T) \propto \Lambda_{1}(T), \\
\lambda_{3}(X)(T) \propto \lambda_{4}(X)(T) \propto \Lambda_{2}(T)
\end{gathered}
$$

supports the second scenario, and

$$
\begin{gathered}
\forall X \exists \Lambda_{1}(T) \exists \Lambda_{2}(T): \\
\lambda_{4}(X)(T) \propto \Lambda_{1}(T), \\
\lambda_{2}(X)(T) \propto \lambda_{3}(X)(T) \propto \Lambda_{2}(T)
\end{gathered}
$$

supports the third scenario.

The second scenario is ruled out immediately, as $T_{F E}$ $<T_{n p t}$ in Table I for the entire class of hexagonal manganites. An order parameter $\lambda_{3}(X)$ of $\sim 0.01 \vec{A}$ (as calculated from the low-temperature structure ${ }^{23-26}$ ) generates only tiny additional peaks to the diffractogram indexed by $P 6_{3} / \mathrm{mmc}$ and would be nearly impossible to detect at $T>1000 \mathrm{~K}$. This rules out the first scenario and leaves us with the third scenario as the only possibility to explain the known data of the transition temperatures as well as the low-temperature structure.

Thus, a comparison of the results from group theory, Fig. 2 , and the known experimental data, Table I, suggests the third scenario depicted in Fig. 2:

$$
K_{3}: P 6_{3} / m m c \Rightarrow P 6_{3} c m
$$

a tilt of the bitetrahedra and a corrugation of the $R$ layers at $T_{n p t}$, followed by

$$
\Gamma_{1}: P 6_{3} \mathrm{~cm} \Rightarrow P 6_{3} \mathrm{~cm}
$$

a ferroelectric distortion of the crystal at $T_{F E}$ for the entire class of hexagonal manganites.

In summary, $P 6_{3} / m m c$ is indeed the high-temperature phase.

\section{SAMPLE PREPARATION AND EXPERIMENTAL DETAILS}

The neutron powder method is ideally suited to probe hexagonal manganites, since $\mathrm{Mn}$ has a fairly large negative scattering length $(b=-3.37 \mathrm{fm})$ and $\mathrm{Tm}, \mathrm{Yb}, \mathrm{Lu}$, and $\mathrm{O}$ have large positive scattering lengths $(7 \leqslant b \leqslant 13 \mathrm{fm}$ and 5.8 $\mathrm{fm})$. Thus, there is a high level of contrast for both cations and oxygen displacements over the $2 \Theta$ range. On the other hand, though contrast of light atoms, e.g. oxygen, is minimal, $\mathrm{x}$-ray diffraction is a fast and readily available method to detect the tripling of the unit cell connected with the symmetry reduction from $P 6_{3} / \mathrm{mmc}$ to $P 6_{3} \mathrm{~cm}$.

For neutron-diffraction experiments large amounts of powder sample were required. The ceramic samples investigated $\left(\mathrm{TmMnO}_{3}, \mathrm{YbMnO}_{3}\right.$, and $\left.\mathrm{LuMnO}_{3}\right)$ were prepared by the solid-state reaction technique at ambient pressure. Cation oxides of $\mathrm{R}_{2} \mathrm{O}_{3}(99.99 \%)$ and $\mathrm{MnO}_{2}$ (99.99\%), ob- 
tained from Alpha Aesar, ${ }^{34}$ were mixed in a 1:2 molar ratio to achieve the stoichiometry of hexagonal $R \mathrm{MnO}_{3}$. The mixture was well pulverized and calcinated in air at $1100^{\circ} \mathrm{C}$ for $24 \mathrm{~h}$. To ensure better homogeneity, the mixtures were ground again and reheated in air at $1000^{\circ} \mathrm{C}$ for $48 \mathrm{~h}$ for all compounds.

After preparation, the samples were checked by $\mathrm{x}$-ray powder diffraction at room temperature to verify the correct hexagonal structure of SG $P 6_{3} \mathrm{~cm}$. Small amounts of $\mathrm{Mn}_{3} \mathrm{O}_{4}$ $(\approx 3-6 \%)$ were found for $\mathrm{LuMnO}_{3}$ after preparation.

Neutron powder-diffraction data were collected on the E9 beam line at the Hahn-Meitner-Institut, Berlin, Germany. Diffractograms were recorded at various temperatures using $10 \mathrm{~g}$ samples mounted in a vanadium can. For hightemperature investigation we have used the high-temperature furnace (HTF), which allowed us to carry out experiments up to $1273 \mathrm{~K}$ and $1373 \mathrm{~K}$ for $\mathrm{TmMnO}_{3}$. Details on the $\mathrm{E} 9$ beam line and spectrometer are given in Ref. 35.

The samples were measured in a $4^{\circ} \leqslant 2 \Theta \leqslant 155^{\circ}$ range with a step size of $0.08^{\circ}$. Effectively, runs lasted around 2-3 $\mathrm{h}$ each for every temperature set point. Temperatures were stabilized within $\pm 5 \mathrm{~K}$. The vanadium can is mounted in the HTF sample chamber which is continuously pumped to avoid oxidation while warming above room temperature. The pressure during all runs was $\approx 10^{-5}$ mbar. We note that changing the pressure and temperature might induce decomposition or loss of oxygen stoichiometry, e.g., $\mathrm{LuMnO}_{3}$ showed small extra reflections after heating to $1273 \mathrm{~K}$ (not shown here), which were associated later with $\mathrm{Lu}_{2} \mathrm{O}_{3}$ by $\mathrm{x}$-ray diffraction at room temperature and electron probe microanalysis.

Furthermore, additional x-ray-diffraction data of $\mathrm{TmMnO}_{3}$ and $\mathrm{YbMnO}_{3}$ were taken at the HTF of the Siemens Bragg-Brentano diffractometer in Leiden, $\lambda$ $=1.54 \AA\left(\mathrm{Cu} K_{\alpha}\right)$, step size $=0.01^{\circ}$, in a $10^{\circ}<2 \Theta<80^{\circ}$ range up to temperatures of $1273 \mathrm{~K}$.

Figure 3 shows x-ray-diffraction data of $\mathrm{YbMnO}_{3}$ at 1273 $\mathrm{K}, 50^{\circ}<2 \Theta<80^{\circ}$. As can be seen clearly, the hightemperature structure $\mathrm{Pb}_{3} / \mathrm{mmc}$ can be ruled out; the structure has to be indexed using a lower symmetry. While a drop of the integrated intensity can be observed with increasing temperature [Fig. 3, bottom, $I(026) / I(032)]$, the large error bars prevent an extrapolation to the transition temperature. For $\mathrm{LuMnO}_{3}$ and $\mathrm{TmMnO}_{3}$, a decrease of intensity of (026) with increasing temperature was also observed, but the peak remained clearly visible for the full temperature range, indicating $T_{n p t}>1300 \mathrm{~K}$.

\section{STATISTICAL EVALUATION OF THE RIETVELD REFINEMENTS}

To obtain the different temperature-dependent order parameters connected to the different modes we refined the neutron powder data using the program SIMREF2.6 (Ref. 36) and the statistical qualifier $N_{\sigma}$ (Ref. 37), taking into account the standard deviation of $2 \Theta$ (Ref. 38), an absorption correction, ${ }^{39,40}$ and an asymmetry correction due to the finite height of the detector. ${ }^{41}$

$N_{\sigma}$ is defined by

$$
N_{\sigma}:=\frac{M-(n-P)}{\sqrt{2(n-P)}}, \quad M:=\sum_{i=1}^{n} \frac{\left(Y_{i}^{o b s}-Y_{i}^{\text {calc }}\right)^{2}}{\sigma_{i}^{2}},
$$

with $n$ being the number of data points, $P$ the number of refined parameters, $Y_{i}^{o b s}$ the observed data points [cts.], $\sigma_{i}$ the standard deviation of observed data points [cts.], and $Y_{i}^{\text {calc }}$ the calculated data points [cts.]. $N_{\sigma}$ itself is obviously random; in an ideal experiment without systematic errors, the expected value of $N_{\sigma}$ and its standard deviation can be estimated by

$$
\begin{gathered}
\forall i \in[1, n]:\left\langle Y_{i}^{\text {obs }}\right\rangle=Y_{i}^{\text {calc }}, \\
\left\langle\sigma_{i}\right\rangle=\sqrt{Y_{i}^{\text {calc }}} \Rightarrow\left\langle N_{\sigma}\right\rangle \approx 0, \quad\left\langle\sigma\left(N_{\sigma}\right)\right\rangle \approx 1 .
\end{gathered}
$$

Thus $N_{\sigma}$ indicates the quality of a fit independent of the number of degrees of freedom $(n-P)$.

As large error bars easily hide a small ferroelectric distortion, we lowered the number of refined parameters by constraining the order parameters and the anisotropic temperature factors $\beta_{i j}$ (Ref. 42) by

$$
\lambda_{3}(\mathrm{Mn})=\lambda_{3}\left(\mathrm{O}_{a p}\right)
$$
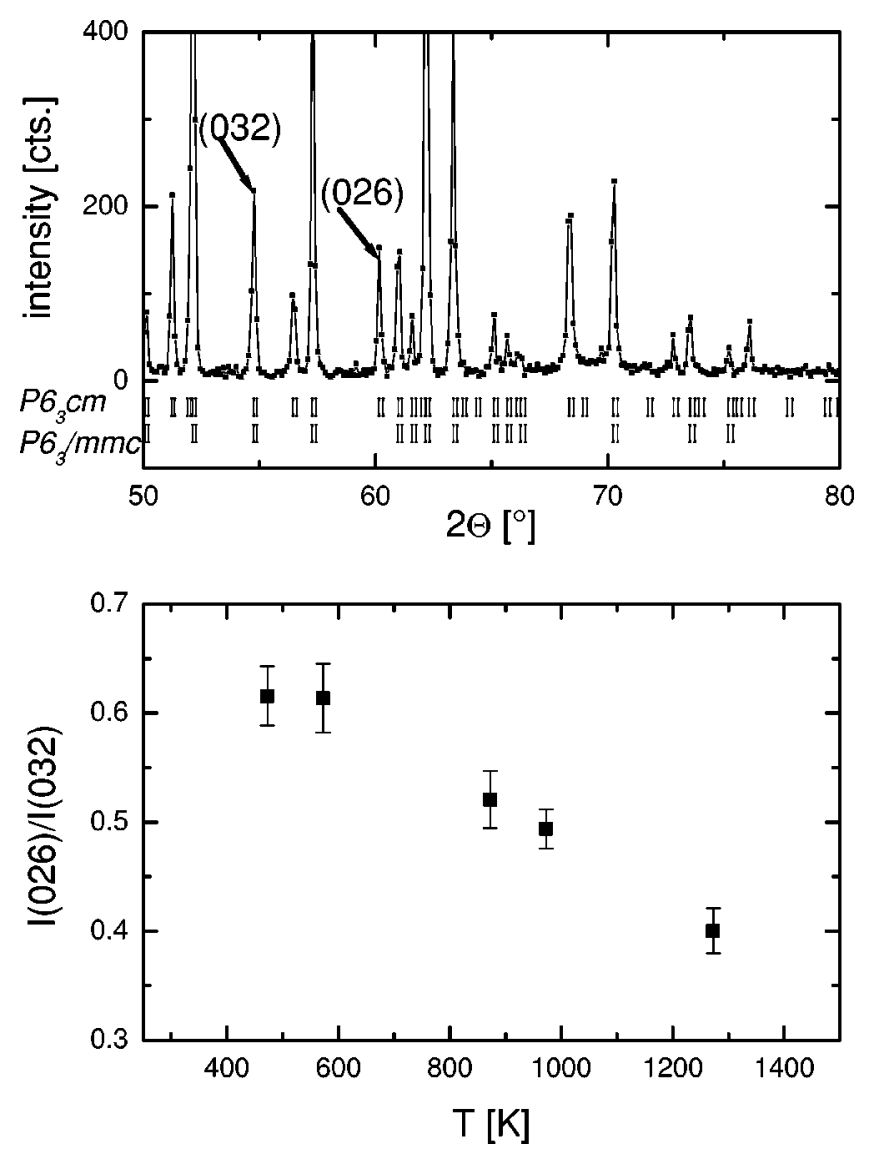

FIG. 3. X-ray powder-diffraction data of $\mathrm{YbMnO}_{3}$ at $1273 \mathrm{~K}$ (top) and integrated intensity (026) normalized by (032) as a function of temperature. The high-temperature structure $P 6_{3} / m m c$ has to be ruled out, a distortion obeying the $K_{3}$ mode is evident and starts to vanish with increasing temperature (see text). 


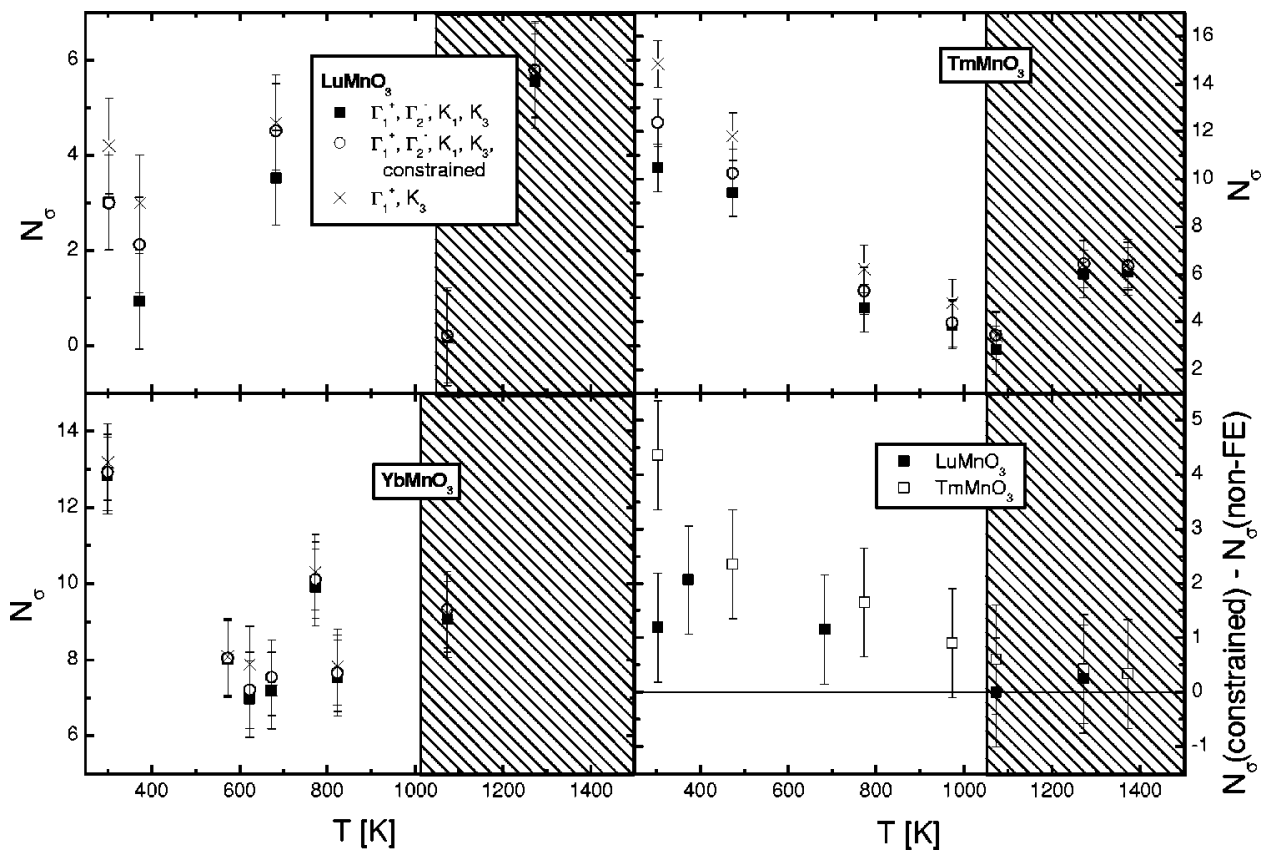

FIG. 4. Statistical qualifiers $N_{\sigma}$ of Rietveld refinements of $R \mathrm{MnO}_{3}$ neutron powder data, $R$ $=\mathrm{Lu}, \mathrm{Tm}, \mathrm{Yb}$. The influence of the proper ferroelectric mode $\Gamma_{1}$ is minimal. Nevertheless a transition temperature of $T_{F E}=1050 \mathrm{~K}$ can be estimated for $R=\mathrm{Tm}$, and $T_{F E}<1100 \mathrm{~K}$ for $R=\mathrm{Lu}$ (lower right). The transition temperature for $R=\mathrm{Yb}$ is taken from Table $\mathrm{I}$. Nonferroelectric regions are shown shaded.

$$
\begin{gathered}
\lambda_{2}(R)=\lambda_{2}\left(\mathrm{O}_{e q}\right), \\
\lambda_{2}\left(\mathrm{O}_{a p}\right)=\lambda_{2}(\mathrm{Mn})=0, \\
\beta_{i j}(R(1))=\beta_{i j}(R(2)), \\
\beta_{i j}(\mathrm{O}(1))=\beta_{i j}(\mathrm{O}(2)), \\
\beta_{i j}(\mathrm{O}(3))=\beta_{i j}(\mathrm{O}(4)) .
\end{gathered}
$$

By this, we explain the spontaneous electric polarization by an in-phase displacement of the $\mathrm{O}(1)-\mathrm{Mn}-\mathrm{O}(2)$ axis parallel to the polar axis $\vec{C}$ : Mn is displaced from the basal plane of the $\mathrm{MnO}_{5}$ bitetrahedron. Indeed the atomic distances $\mathrm{Mn}-\mathrm{O}_{e q}$ and $\mathrm{Mn}-\mathrm{O}_{a p}$ compared to $R-\mathrm{O}_{a p}$ in the hightemperature phase $^{19}$ give further support to the approach of a displacement of the $\mathrm{O}(1)-\mathrm{Mn}-\mathrm{O}(2)$ axis: the distances between $\mathrm{Mn}$ and the equatorial oxygen positions tend to be far larger $(\approx 2.1 \AA)$ than between $\mathrm{Mn}$ and the apical positions $(\approx 1.85 \AA)$. The latter are comparable to the sum of the added ionic diameters, ${ }^{43}$ as well as the atomic distances between $R$ and the apical oxygen positions $R-\mathrm{O}_{a p}$. We propose that a thermal stabilization of $\mathrm{Mn}$ in the basal plane of the $\mathrm{MnO}_{5}$ bitetrahedron is removed in a soft-mode transition by lowering the temperature, leading to a spontaneous electric polarization. The displacement of the apical oxygen positions accompanying the Mn displacement is directly explained by the small $\mathrm{Mn}-\mathrm{O}_{a p}$ distance.

Figure 4 shows the statistical qualifiers of Rietveld refinements of $R \mathrm{MnO}_{3}$ powder-diffraction data, $R=\mathrm{Lu}, \mathrm{Tm}$, and Yb. Obviously the effect of a refinement of $\lambda_{2}$ is minimal: refinements using the constraints (20)-(25) result in qualifiers comparable to those of the approach without constraints in the full temperature region. For $R=\mathrm{Lu}$ and Tm qualifiers of refinements without $\lambda_{2}$ but with the constraints (20) and (23) $-(25)$ tend to increase (lower right). For $R=\mathrm{Yb}$, a comparison of the qualifiers remains ambiguous. Based on the comparison of the statistical qualifiers of the refinements of the different models, we propose transition temperatures of $T_{F E}=1050(50) \mathrm{K}$ for $R=\mathrm{Tm}$ and of $T_{F E}<1100 \mathrm{~K}$ for $R$ $=\mathrm{Lu}$. The transition temperature $T_{F E}$ for $R=\mathrm{Yb}$ is taken from Table I. Nonferroelectric regions are shown shaded in Figs. 4-6.

Figure 5 displays the results of our refinements of the $\mathrm{TmMnO}_{3}$ powder-diffraction data using the constraints (20)(25): the order parameters $\lambda_{2}, \lambda_{3}$, and $\lambda_{4}$, the crystal axes and the anisotropic temperature factors of $\mathrm{Mn}$, the apical oxygen positions, Tm and the equatorial oxygen positions. The order parameters $\lambda_{4}(X)$ are normalized to $\lambda \Rightarrow 1$ for $T \Rightarrow 0 \mathrm{~K}$. For clarity, the tilting angles $\varphi_{a p}$ and $\varphi_{e q}$,

$$
\begin{gathered}
\tan \left(\varphi_{a p}\right)=\frac{\lambda_{4}\left(\mathrm{O}_{a p}\right) A}{z(\mathrm{O}(1)) C}, \\
\tan \left(\varphi_{e q}\right)=\frac{\frac{3}{2} \lambda_{4}\left(\mathrm{O}_{e q}\right) C}{\frac{1}{2} A}=\frac{3 \lambda_{4}\left(\mathrm{O}_{e q}\right) C}{A},
\end{gathered}
$$

of the $\mathrm{O}(1)-\mathrm{Mn}-\mathrm{O}(2)$ axis and of the basal plane of the $\mathrm{MnO}_{5}$ bitetrahedron are depicted additionally in Fig. 6, instead of the order parameters of $\lambda_{4}\left(\mathrm{O}_{a p}\right)$ and $\lambda_{4}\left(\mathrm{O}_{e q}\right)$. As the $z$ position of $\mathrm{O}_{e q}$ stays constant within the error bars $\left(\lambda_{1} \approx 0\right.$, not shown here) and changes in the ratio of the axes are minimal, Fig. 6 is in fact equivalent to the depiction of $\lambda_{4}(X)$ in Fig. 5. Anisotropic temperature factors, which are not shown here, are either constrained by the symmetry of the SG or zero within the error bars of our Rietveld refinements.

Though the order parameter $\lambda_{3}$ remains ambiguous within the error bars, we propose tentatively $\lambda_{2} \propto \lambda_{3}$, with a transi- 

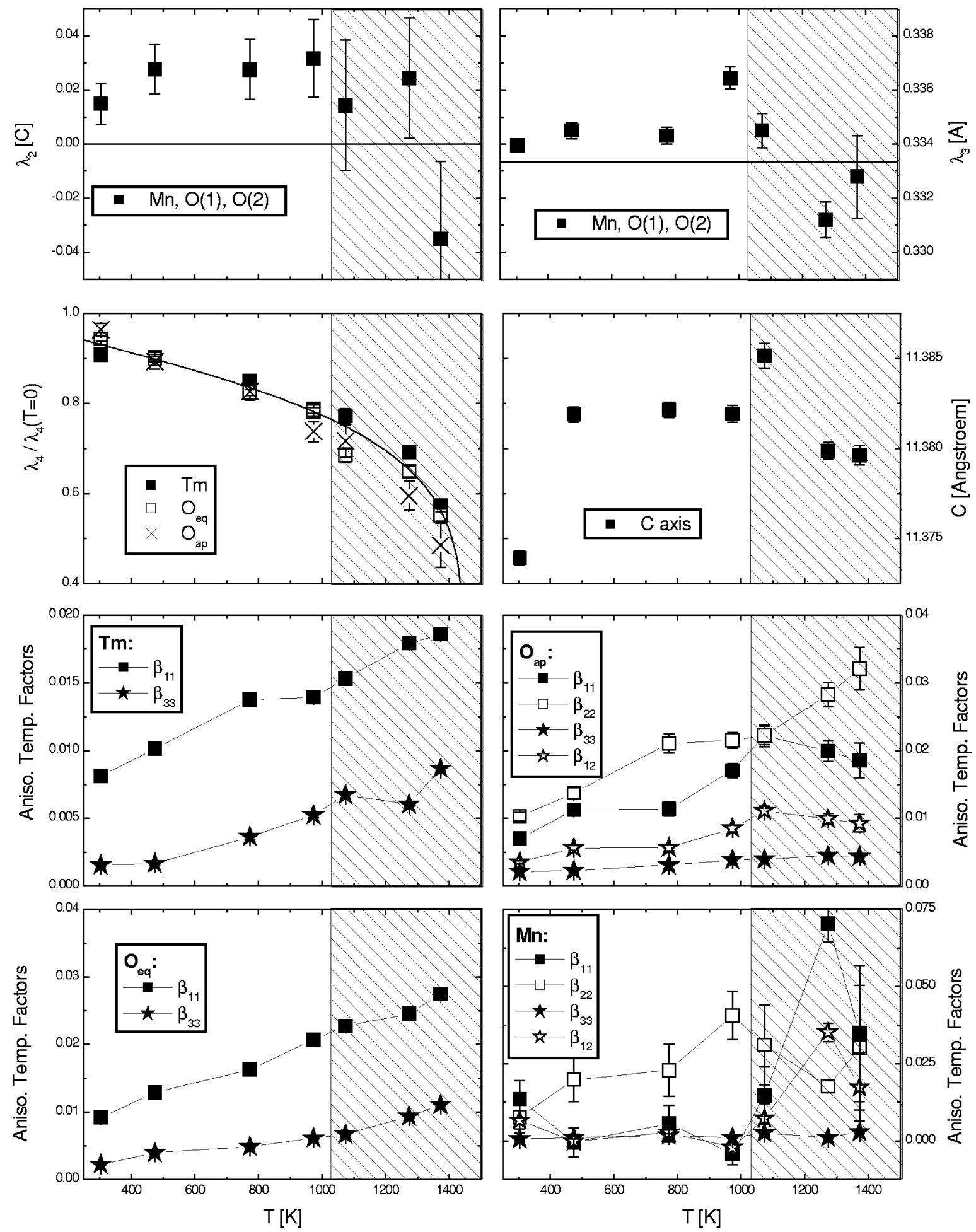

FIG. 5. Temperature dependence of order parameters, $C$ axis, and anisotropic temperature factors of $\mathrm{TmMnO}_{3}$. The order parameter $\lambda_{4}(X)$ is normalized to $\lambda_{4} \Rightarrow 1$ for $T \Rightarrow 0 \mathrm{~K}$. A fit to an empirical formula (Ref. 44) gives clear evidence for the common origin of the displacement of oxygen and $R$ due to the $K_{3}$ mode. A kink in the $C$ axis and kinks in the anisotropic temperature factors of Mn and the apical oxygen positions give evidence for the onset of a spontaneous electric polarization at $T \approx 1050(50) \mathrm{K}$, due to a soft-mode transition, while the development of the anisotropic temperature factors of $\mathrm{Tm}$ and the equatorial oxygen positions remain nearly unaffected. This agrees with $\lambda_{2}$ vanishing in the error bars. $\lambda_{3}$ remains ambiguous due to low statistics, but is connected to $\lambda_{2}$ by group theory. The nonferroelectric regions are shown shaded. 


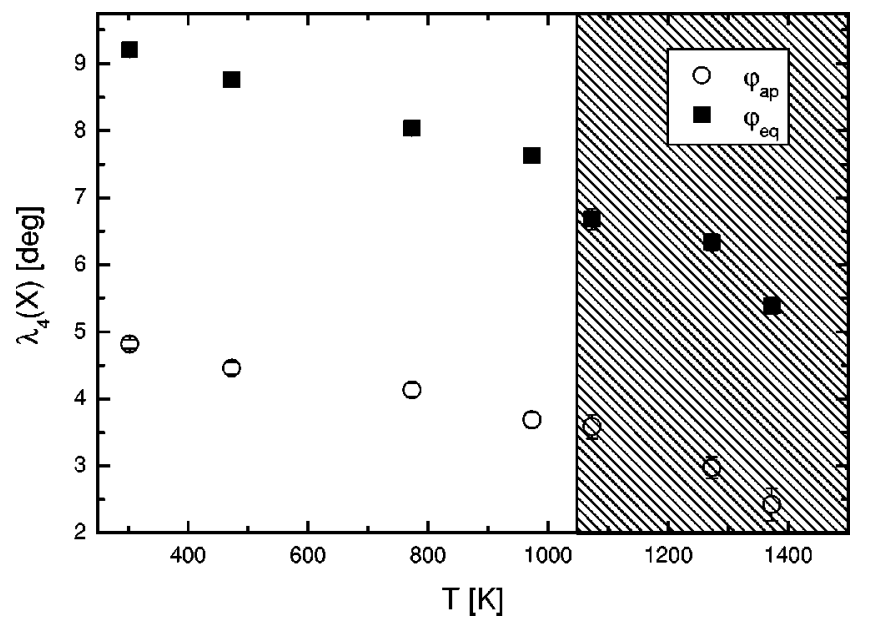

FIG. 6. Two order parameters of the improper ferroelectric mode $K_{3}$ depicted as tilting angles: with increasing temperature the tilt gets smaller while $\varphi_{a p} \approx 1 / 2 \varphi_{e q}$. The nonferroelectric region is shown shaded.

tion temperature of $T_{F E}$, as group theory suggests only one transition temperature (Fig. 2).

While obviously the $K_{3}$ mode is active and the unit cell is tripled in the full temperature range, the values of the three order parameters of the $K_{3}$ mode decrease clearly with increasing temperature, keeping a constant ratio, as can be seen in Fig. 5. Using a fit with the empirical formula ${ }^{44}$

$$
\lambda_{4}(X)=\lambda_{4}(X)(T=0)\left[1-\left(\frac{T}{T_{n p t}}\right)^{\alpha}\right]^{\beta},
$$

with $\Lambda_{2}(X)(T=0)$ and $\alpha, \beta$ free parameters, the three order parameters $\lambda_{4}(X)$ can be normalized to the order parameter

$$
\Lambda_{2}(T)=\frac{1}{\lambda_{4}(X)(T=0)} \lambda_{4}(X)(T),
$$

fit parameters are shown in Table V. Obviously the order parameter $\lambda_{4}(X)$ obeys the same temperature dependence, giving clear evidence for the common origin of the displacement of oxygen and $R$ due to the $K_{3}$ mode, while a common temperature dependence of $\lambda_{2}(X)$ and $\lambda_{3}(X)$ cannot be excluded.

In contrast, the anisotropic temperature factors of the apical oxygen atoms and Mn show clearly a symmetry change of the temperature movement at $\approx 1050(50) \mathrm{K}$, e.g., com-

TABLE V. Fit parameters of the order parameter $\Lambda_{2}$.

\begin{tabular}{lccc}
\hline \hline$\lambda_{4}(R)_{T=0}(\mathrm{C})$ & $\lambda_{4}\left(\varphi_{a p}\right)_{T=0}(\mathrm{deg})$ & $\lambda_{4}\left(\varphi_{e q}\right)_{T=0}(\mathrm{deg})$ & $\alpha$ \\
$0.02123(26)$ & $4.3(11)$ & $8.8(22)$ & $1.35(37)$ \\
\hline$\beta$ & $T_{n p t}(\mathrm{~K})$ & $\chi^{2}$ & \\
$0.168(32)$ & $1433(27)$ & 0.41 & \\
\hline \hline
\end{tabular}

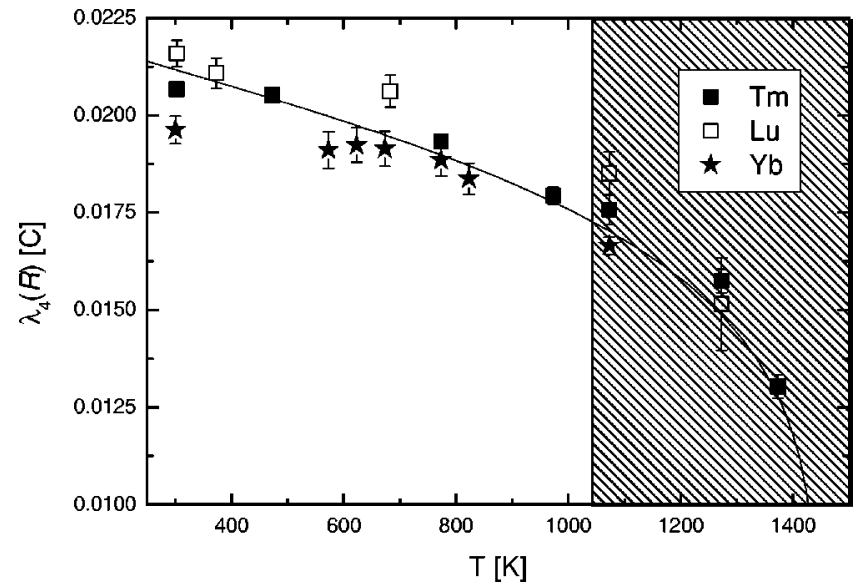

FIG. 7. Temperature dependence of the order parameters $\lambda_{4}(R)$, $R=\mathrm{Lu}, \mathrm{Tm}, \mathrm{Yb}$. A fit of $\lambda_{4}(\mathrm{Tm})$ to Eq. (28) for $R=\mathrm{Tm}$ is added as a guide to the eye. The nonferroelectric region is shown shaded.

pare $\beta_{11}$ and $\beta_{22}$, while the polar $C$ axis shows a kink and the ferroelectric distortion becomes nonsignificant within statistics at the same temperature. Again, this gives further evidence for the activation of the proper ferroelectric $\Gamma_{1}$ mode at $\approx 1050(50) \mathrm{K}$ and indicates a soft-mode transition. The anisotropic temperature factors of $\mathrm{O}_{e q}$ and $\mathrm{Tm}$ remain nearly unaffected; the ferroelectric mode $\Gamma_{1}$ : $P 6_{3} \mathrm{~cm} \Rightarrow P 6_{3} \mathrm{~cm}$ displaces mainly the $\mathrm{O}(1)-\mathrm{Mn}-\mathrm{O}(2)$ axis of the $\mathrm{MnO}_{5}$ bitetrahedron.

As can be seen in Fig. 7, the temperature-dependent order parameter $\lambda_{4}(R), R=\mathrm{Lu}$, Tm, Yb shows comparable behavior for the three measured $R \mathrm{MnO}_{3}$ compounds. As the distortions are clearly visible, but cannot lead to a spontaneous electric polarization below $T=T_{F E}$ (14), hexagonal manganites must be described as triangular antiferroelectric for $T_{F E}<T<T_{n p t}$ and as triangular ferroelectric, or in analogy to magnetism, canted antiferroelectric, for $T<T_{F E}$. With the knowledge of the magnetic ordering process, ${ }^{3,13,14}$ the transition temperatures of Table I and our results, we propose a schematic phase diagram for the entire class of hexagonal manganites (Fig. 8). Figure 9 shows the three phases, the modes, and the displacements of the atomic positions.

\section{SUMMARY}

We presented calculations of the irreducible representations for the low-temperature phase of hexagonal manganites, resulting in four different orthogonal modes and order parameters. A comparison of our calculations to published experimental data ${ }^{19-26}$ indicates a two-step phase transition $P 6_{3} / m m c \Rightarrow P 6_{3} c m \Rightarrow P 6_{3} c m$ at temperature $T_{n p t}>T_{F E}$, in particular, the existence of the aristotype at high temperatures is shown. By x-ray and neutron powder diffraction the low-temperature symmetry of $\mathrm{Pb}_{3} \mathrm{~cm}$ was verified for $R$ $=\mathrm{Lu}, \mathrm{Tm}, \mathrm{Yb}$ over the full temperature range $R T<T$ $<1273 \mathrm{~K}$ and up to $1373 \mathrm{~K}$ for $\mathrm{TmMnO}_{3}$. Rietveld refinements of the order parameters of neutron powder data indi- 


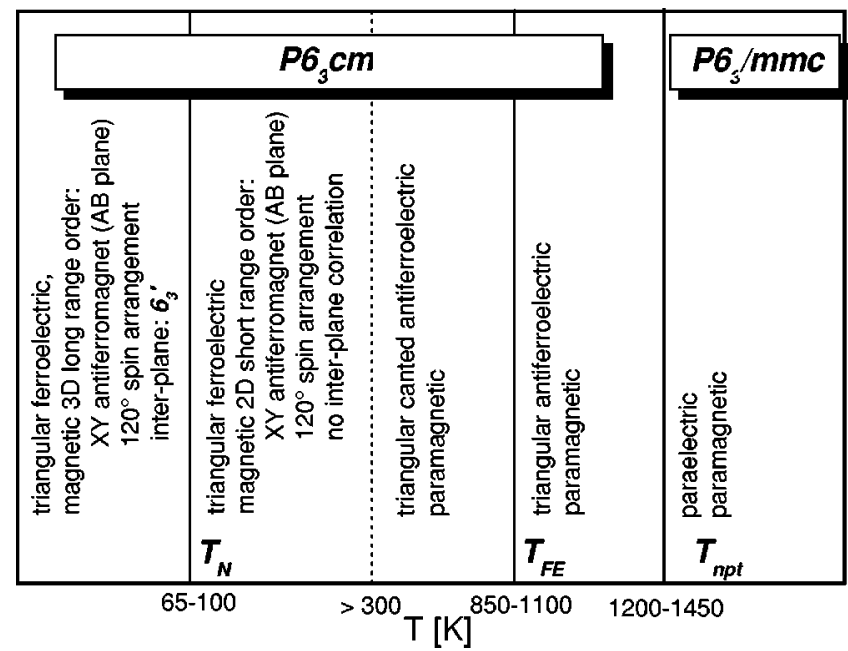

FIG. 8. Schematic phase diagram of electric and magnetic ordering processes in hexagonal manganites, based on Refs. 3, 13, and 14, Table I, and this paper. Transition temperatures vary with different $R$.

cated a soft-mode transition at a temperature of $T_{F E}$ $=1050(50) \mathrm{K}$ for $R=\mathrm{Lu}, \mathrm{Tm}$, as the $\mathrm{O}(1)-\mathrm{Mn}-\mathrm{O}(2)$ axis is displaced from the basal plane of the $\mathrm{MnO}_{5}$ bitetrahedron. This generates a spontaneous electric polarization. A nuclear transition temperature at $T_{n p t}=1433(27) \mathrm{K}$, connected with a tripling of the unit cell by a tilt of the $\mathrm{MnO}_{5}$ bitetrahedra and a buckling of the $R$ layers, was extrapolated by an empiric formula for $R=\mathrm{Tm}$. For $R=\mathrm{Lu}, \mathrm{Yb}$ similar transition temperatures can be estimated. This allows us to present a schematic phase diagram of electric and magnetic ordering processes in hexagonal manganites. Our results are in full

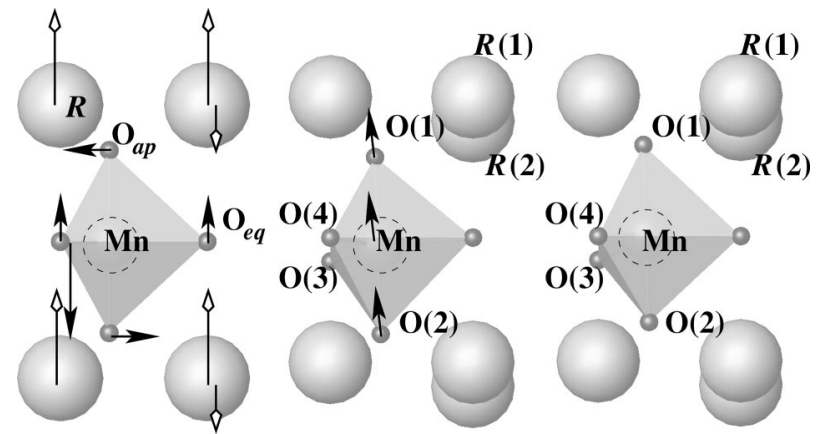

FIG. 9. Local coordination of the $\mathrm{MnO}_{5}$ bitetrahedron in the $A C$ plane. Hexagonal manganites order in three nuclear phases: paraelectric $P 6_{3} / m m c$ for $T>T_{n p t}$ (left), triangularantiferroelectric $P 6_{3} \mathrm{~cm}$ for $T_{n p t}>T>T_{F E}$ (middle) and triangularferroelectric $P 6_{3} \mathrm{~cm}$ for $T_{F E}>T$ (right). The paraelectric to triangular-antiferroelectric phase transition at $T_{n p t}$ is described by a $K_{3}: P 6_{3} / m m c \rightarrow P 6_{3} c m$ mode; the mode tilts the $\mathrm{MnO}_{5}$ bitetrahedron (black arrows) and corrugates the $R$ layer (white arrows). The triangular-antiferroelectric to ferroelectric phase transition at $T_{F E}$ is described by a $\Gamma_{1}: P 6_{3} \mathrm{~cm} \rightarrow P 6_{3} \mathrm{~cm}$ mode. The $\mathrm{O}(1)-\mathrm{Mn}-\mathrm{O}(2)$ axis is displaced and a spontaneous electric polarization is generated. The arrows indicate the direction of the displacements. Note that the displacement of the $\mathrm{O}(1)-\mathrm{Mn}-\mathrm{O}(2)$ axis due to the $\Gamma_{1}$ : $P 6_{3} \mathrm{~cm} \rightarrow P 6_{3} \mathrm{~cm}$ mode is slightly tilted against the $C$ axis.

agreement with both our group-theoretical calculations and published experimental data ${ }^{19-26}$ of hexagonal manganites.

\section{ACKNOWLEDGMENTS}

The authors acknowledge the financial support of the German BMBF (Grant No. 03PRE7TU) and the Dutch foundation FOM.
*Electronic address: thomas.lonkai@uni-tuebingen.de

†Electronic address: tomuta@phys.leidenuniv.nl

${ }^{1}$ See, for example, Z.J. Huang, Y. Cao, Y.Y. Sun, Y.Y. Xue, and C.W. Chu, Phys. Rev. B 56, 2623 (1997).

${ }^{2}$ E.F. Bertaut and M. Mercier, Phys. Lett. 5, 27 (1963).

${ }^{3}$ T. Lonkai, D.G. Tomuta, J.U. Hoffmann, R. Schneider, D. Hohlwein, and J. Ihringer, J. Appl. Phys. 93, 8191 (2003).

${ }^{4}$ M. Fiebig, T. Lottermoser, D. Fröhlich, and R.V. Pisarev, Nature (London) 419, 818 (2002).

${ }^{5}$ A.V. Goltsev, R.V. Pisarev, T. Lottermoser, and M. Fiebig, Phys. Rev. Lett. 90, 177204 (2003).

${ }^{6}$ N.F.D. Ito, T. Yoshimura, and T. Ito, J. Appl. Phys. 93, 5563 (2003).

${ }^{7}$ N. Fujimura, H. Sakata, D. Ito, T. Yoshimura, T. Yokota, and T. Ito, J. Appl. Phys. 93, 6990 (2003).

${ }^{8}$ H.L. Yakel, W.C. Koehler, E.F. Bertaut, and E.F. Forrat, Acta Crystallogr. 16, 957 (1963).

${ }^{9}$ T. Katsufuji, S. Mori, M. Masaki, Y. Moritomo, N. Yamamoto, and H. Takagi, Phys. Rev. B 64, 104419 (2001).

${ }^{10}$ D.G. Tomuta, S. Ramakrishnan, G.J. Nieuwenhuys, and J.A. Mydosh, J. Phys.: Condens. Matter 13, 4553 (2001).

${ }^{11}$ K. Yoshii and H. Abe, J. Solid State Chem. 165, 131 (2002).

${ }^{12}$ A. Muñoz, J.A. Alonso, M.J. Martínez-Lope, M.T. Casiás, J.L.
Martínez, and M.T. Fernández-Díaz, Phys. Rev. B 62, 9498 (2000).

${ }^{13}$ M. Fiebig, D. Fröhlich, K. Kohn, S. Leute, T.L.V.V. Pavlov, and R.V. Pisarev, Phys. Rev. Lett. 84, 5620 (2000).

${ }^{14}$ T. Lonkai, D. Hohlwein, J. Ihringer, and W. Prandl, Appl. Phys. A: Mater. Sci. Process. 74, S843 (2002).

${ }^{15}$ A. Filippetti and N.A. Hill, Phys. Rev. B 65, 195120 (2002).

${ }^{16}$ J.E. Medvedeva, V.I. Anisimov, M.A. Korotin, O.N. Mryasov, and A.J. Freeman, J. Phys.: Condens. Matter 12, 4947 (2000).

${ }^{17}$ A. Filippetti and N.A. Hill, Phys. Rev. B 67, 125109 (2003).

${ }^{18}$ T. Katsufuji, S. Mori, M. Masaki, Y. Moritomo, N. Yamamoto, and H. Takagi, Phys. Rev. B 66, 134434 (2002).

${ }^{19}$ S.C. Abrahams, Acta Crystallogr., Sect. B: Struct. Sci. B57, 485 (2001).

${ }^{20}$ K. Lukaszewicz and J. Karat-Kalicinska, Ferroelectrics 7, 81 (1974).

${ }^{21}$ I.G. Izmailzade and A. Kizhaev, Sov. Phys. Solid State 7, 236 (1965).

${ }^{22}$ G.A. Smolenskii and I.E. Chupis, Sov. Phys. Usp. 25, 475 (1982), and references therein.

${ }^{23}$ B.B. van Aken, A. Meetsma, and T.T.M. Palstra, Acta Crystallogr., Sect. C: Cryst. Struct. Commun. C57, 230 (2001).

${ }^{24}$ B.B. van Aken, A. Meetsma, and T.T.M. Palstra, Acta Crystal- 
logr., Sect. E: Struct. Rep. Online 57, 38 (2001).

${ }^{25}$ B.B. van Aken, A. Meetsma, and T.T.M. Palstra, Acta Crystallogr., Sect. E: Struct. Rep. Online 57, 87 (2001).

${ }^{26}$ B.B. van Aken, A. Meetsma, and T.T.M. Palstra, Acta Crystallogr., Sect. E: Struct. Rep. Online 57, 101 (2001).

${ }^{27}$ S. Geller, J.B. Jeffries, and P.J. Curlander, Acta Crystallogr., Sect. B: Struct. Crystallogr. Cryst. Chem. B31, 2770 (1975).

${ }^{28}$ H. Megaw, Crystal Structures: A Working Approach (Saunders, Philadelphia, 1973).

${ }^{29}$ H.T. Stokes and D.M. Hatch, 2002, ISOTROPY, http:// www.physics.byu.edu/ stokesh/isotropy.html

${ }^{30}$ H.T. Stokes and D.M. Hatch, Isotropy Subgroups of the 230 Crystallographic Space Groups (World Scientific, Singapore, 1988).

${ }^{31}$ To avoid complex distortion fields, we take the atomic positions from the fifth column of Table II, write the displacements in $\langle\vec{A}, \vec{B}, \vec{C}\rangle$ and use the symmetry operations of $\mathrm{PG}_{3} \mathrm{~cm}$ to obtain displacements of symmetrically equivalent positions.

${ }^{32}$ Note that the displacements of Mn parallel $z$ can be nonzero, but, as $P 6_{3} \mathrm{~cm}$ is a polar structure, the $z$ coordinate of $\mathrm{Mn}$ is usually fixed to zero. Thus a displacement $\lambda\left(\Gamma_{2}^{-}\right)(\mathrm{Mn})$ is usually calculated as a displacement of $-\lambda\left(\Gamma_{2}^{-}\right)(\mathrm{Mn})$ of all other atomic positions.

${ }^{33}$ Note that $\Gamma_{1}^{+}: \quad P 6_{3} / m m c \rightarrow P 6_{3} / m m c$ and $\Gamma_{1}: P 6_{3} c m$ $\rightarrow P 6_{3} \mathrm{~cm}$ are different modes. After the first phase transition
$K_{3}: P 6_{3} / m m c \rightarrow P 6_{3} \mathrm{~cm}$, the symmetry of the system has changed, and the modes have to be recalculated.

${ }^{34}$ Alfa Aesar Johnson Matthey GmbH, 76057 Karlsruhe, Germany, dcat@alpfa.com

${ }^{35}$ D.M. Többens, N. Stüser, K. Knorr, H.M. Mayer, and G. Lampert, Mater. Sci. Forum 378-381, 288 (2001).

${ }^{36}$ H. Ritter, J. Ihringer, J.K. Maichle, and W. Prandl, 1999, SIMREF2.6, http://www.uni-tuebingen.de/uni/pki/simref/simRef. html

${ }^{37}$ J. Ihringer, J. Appl. Crystallogr. 28, 618 (1995).

${ }^{38}$ C. Rebmann, H. Ritter, and J. Ihringer, Acta Crystallogr., Sect. A: Found. Crystallogr. A54, 225 (1998).

${ }^{39}$ K.D. Rouse and M.J. Cooper, Acta Crystallogr., Sect. A: Cryst. Phys., Diffr., Theor. Gen. Crystallogr. A26, 682 (1970).

${ }^{40}$ A.W. Hewat, Acta Crystallogr., Sect. A: Cryst. Phys., Diffr., Theor. Gen. Crystallogr. A35, 248 (1979).

${ }^{41}$ J.F. Bérar and G. Baldinozzi, J. Appl. Crystallogr. 26, 128 (1993).

${ }^{42}$ The anisotropic movement of the ions in the crystal leads to a correction term for the peak intensity of peak $(h k l)$ of $\exp \left[-\left(\beta_{11} h^{2}+\beta_{22} k^{2}+\beta_{3} l^{2}+\beta_{12} h k+\beta_{13} h l+\beta_{23} k l\right)\right]$.

${ }^{43}$ R.D. Shannon, Acta Crystallogr., Sect. A: Cryst. Phys., Diffr., Theor. Gen. Crystallogr. A32, 751 (1976).

${ }^{44}$ K. Hagdorn, D. Hohlwein, J. Ihringer, K. Knorr, W. Prandl, H. Ritter, H. Schmid, and T. Zeiske, Eur. Phys. J. B 11, 243 (1999). 